\title{
Bibliographical references
}


This page is intentionally left blank

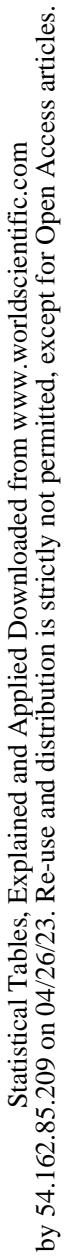


Recent and easily accessed references were preferred for this short bibliography; we make exceptions for references to original papers in cases of the less documented distributions.

BARLOW, R.E., BARTHOLOMEW, D.J., BREMNER, J.M., BRUNK, H.D. (1972). Statistical inference under order restrictions. New York, Wiley. [ $\left[\overline{\mathrm{E}}^{2}\right.$ for monotonic variation] BRATLEY, P. FOX, B.L., SCHRAGE, L.E. (1987). A guide to simulation (2 ${ }^{\text {nd }}$ ed.). New York, Springer-Verlag. $[F]$

COCHRAN, W.G. (1941). The distribution of the largest of a set of estimated variances as a fraction of their total. Annals of Eugenics, 11, 47-52. [Cochran's C]

DAVID, H.A. (1952). Upper 5 and $1 \%$ of the maximum $F$-ratio. Biometrika, 39, 422-424. $\left[F_{\max }\right]$

DEVROYE, L. (1986). Non-uniform random variate generation. New York, Springer-Verlag. [General, notably Normal distr.]

DUNNETT, C.W. (1955). A multiple comparison procedure for comparing several treatments with a control. Journal of the American statistical association, 50, 1096-1121. [Dunnett's $t$ ]

FISHER, R.A. (1970). Statistical methods for research workers $\left(14^{\text {th }}\right.$ ed.). New York, Hafner. [General, notably Orthogonal polyynomials]

FISHMAN, G.S. (1996). Monte Carlo : concepts, algorithms, and applications. New York, Springer-Verlag. $\left[\chi^{2}\right]$

GENTLE, J.E. (1998). Random number generation and Monte Carlo methods. New York, Springer-Verlag. [Normal, $t$, Binomial, Random numbers]

GERALD, C.F., WHEATLEY, P.O. (1984). Applied numerical analysis (3 ${ }^{\text {rd }}$ ed.). Reading (MA), Addison-Wesley. [Mathematical complements: Numerical integration]

HARTLEY, H.O. (1950). The maximum $F$-ratio as a short-cut test for heterogeneity of variance. Biometrika, 37, 308-312. $\left[F_{\max }\right]$

HOCHBERG, Y., TAMHANE, A.C. (1987). Multiple comparison procedures. New York, Wiley. [Studentized range, Dunnett's $t$ ]

JOHNSON, N.L., KOTZ, S., BALAKRISHNAN, N. (1994, 1995). Continuous univariate distributions $\left(2^{\text {nd }}\right.$ ed.), 2 volumes. New York, Wiley. [Normal, $\left.\chi^{2}, t, F, B e ̂ t a\right]$ 
JOHNSON, N.L., KOTZ, S., KEMP, A.W. (1992). Univariate discrete distributions $\left(2^{\text {nd }}\right.$ ed.). New York, Wiley. [Binomial, Number of runs]

KENDALL, M.G., STUART, A. (1977). The advanced theory of statistics. Vol. 1: Distribution theory $\left(4^{\text {th }}\right.$ ed.). New York, Macmillan. [General, including Mathematical complements]

KNUTH, D.E. (1969). The art of computer programming. Vol. 2: Seminumerical algorithms. Reading (MA), Addison-Wesley. [Random numbers]

LAURENCELLE, L. (1993). Deux tests de variation monotone pour l'analyse de variance. Lettres Statistiques, 9, 69-91. [ $\overline{\mathrm{E}}^{2}$ for monotonic variation]

LAURENCELLE, L. (1995). Le nombre total de suites: moments, approximations et valeurs critiques. Actes du Colloque sur les Méthodes et Applications de la Statistique 1995, p. 73-88. Sainte-Foy, Bureau de la statistique du Québec. [Number of runs]

LAURENCELLE, L. (1998). Théorie et techniques de la mesure instrumentale. Sainte-Foy, Canada, Presses de l'Université du Québec. [Supplementary example $n^{\circ} 5$ ]

LAURENCELLE, L. (2000). Le quotient de deux variances corrélées. Lettres Statistiques, 11, 15-26. $[F]$

LAURENCELLE, L. (2001). Hasard, nombres aléatoires et méthode Monte Carlo. Sainte-Foy, Canada, Presses de l'Université du Québec. [General, Random numbers]

MYERS, J.L. (1979). Fundamentals of experimental design ( $3^{\text {rd }}$ ed.). Boston, Allyn and Bacon. [Orthogonal polynomials]

OWEN, D.B. (1962). Handbook of statistical tables. Reading (MA), Addison-Wesley. [General] WINER, B.J. (1971, $2^{\text {nd }}$ ed.; 1991, $3^{\text {rd }}$ ed., with D.R. BROWN \& M.K. MICHELS). Statistical principles in experimental design. New York, McGraw-Hill. [General, notably Orthogonal polynomials] 


\section{Index of examples sorted by type of distribution}

Normal distribution

Normal score interval comprising central $50 \%$ of the population

Proportion (number of elements) in a population (with Normal distribution) located over a given value

Test for the difference between measures (or means) of two objects (or groups) using the standard error of measurement (or standard deviation of population)

Test for a sample mean, with population variance $\left(\sigma^{2}\right)$ known

Test for a correlation coefficient $\left(\mathrm{H}_{0}: \rho \neq 0\right)$

Test for the difference of means between two groups

Test for the difference between two independent correlation coefficients

\section{Chi-square distribution $\left(\chi^{2}\right)$}

Confidence interval for a variance

Test of independence (or interaction) in a two-way frequency table Test of goodness of fit

Test for the difference among $k$ independent variances (Bartlett's test on homogeneity of variances)
Section Page

Normal 1

Normal $2 \quad 12$

Normal 3

12

Supp. 1

185

Supp. $3 \quad 186$

Supp. $6 \quad 189$

Supp. 9

191

$\chi^{2} 1$

$\chi^{2} 2$

Supp. 5

Supp. 10

192 
Student's $t$ distribution

Confidence interval for a mean

Test for the difference of means between two (independent) groups

Planned tests for the differences between means following Dunn-Šidák's criterion

Test for a correlation coefficient $\left(\mathrm{H}_{0}: \rho=0\right)$

Test for a sample, with population variance $\left(\sigma^{2}\right)$ unknown

Confidence interval for a predicted value based on linear regression

Test for the difference between two paired means (or between means of paired groups)

Test for the difference between two paired variances (or between variances of paired groups)

\section{$F$ distribution}

Global $F$ test in analysis of variance (ANOVA) for $k$ independent $\quad F 1$ groups

Test for the difference between two independent variances (homogeneity $\quad F 2$ test for two variances)

Test for significant powers of the regressor in linear polynomial OP 1 regression with $k$ levels

Tests on a set of contrasts among means, according to Scheffé's criterion

\section{Studentized range (q) distribution}

Post hoc tests for the differences between means following Tukey's HSD criterion

Post hoc tests for the differences between means following Newman-
Section Page 35 


\section{Dunnett's $t$ distribution}

Test for the differences between each of $p-1$ means and a reference mean (Dunnett's test)

\section{$\overline{\mathrm{E}}^{2}$ distribution for monotonic variation}

Test of the hypothesis of simple order in monotonic variation for $k$ means

Test of the hypothesis of dominance (or simple tree order) in monotonic variation for $k$ means

\section{$F_{\text {max }}$ distribution}

Test for the difference between $k$ independent variances (Hartley's test for homogeneity of variances), with equal $d f$ 's

Test for the difference between $k$ independent variances (Hartley's test $\quad F_{\max } 2$ for homogeneity of variances), with unequal $d f$ 's

\section{Cochran's $C$ distribution}

Test for the difference between $k$ independent variances (Cochran's test for homogeneity of variances), with equal $d f$ 's

Test for the difference between $k$ independent variances (Cochran's test for homogeneity of variances), with unequal $d f$ 's

\section{Orthogonal polynomials (OP)}

Determination of significant powers of the regressor variable in linear orthogonal regression for $k$ means, and reconstruction of a regular polynomial equation

Simple linear regression with orthogonal polynomials

Direct computation of $1^{\text {st }}$ and $2^{\text {nd }}$ degree coefficients for any set $(\mathrm{N})$ of 
Binomial distribution (Bin)

Section Page

Computation of probability of success in blind answering of a multiple-

Bin 1

152 choice multi-item exam

Confidence interval for a proportion or percentage

Bin 2

152

Test of the hypothesis of equal probabilities for a series of observations

Bin 3

with two categories

Computations, exact and approximate, of a binomial probability

Bin 4

153

Number-of-runs (r) distribution

Test of randomness for a sequence of objects of two types in a queue

$r 1$

171

Test of randomness for the sequence of residuals $\left(\mathrm{Y}_{i}-\widehat{\mathrm{Y}}_{i}\right)$ in linear

$r 2$

172 regression 


\section{General index}

Analysis of variance (ANOVA) one-way, $k$ equal groups 54678196 103133208

unequal groups (test for homogeneity of variances) 208

Approximation

to binomial by normal $163165-167$

to Chi-square by normal 2124

to $F$ by normal 5459

to number-of-runs by normal 184

to Student's $t$ by normal 40

Beta distribution 213 function 213

Binomial expansion 164213 and combinations 213

and d.f. of Student's $t 38$

Bonferroni criterion

see Dunn-Šidák criterion

Combinations 214

and binomial expansion 213
Confidence interval

for diff. between two error-prone measurements 12

for a sample mean 36

for a predicted value from linear regression 201

for a proportion (percentage) 163

for a sample variance (and s.d.) 21

Contrasts

see Test procedures

Correlation Def. $\left(\rho_{\mathrm{XY}}, r_{\mathrm{XY}}\right) 214$ between contrasts in Dunnett's method 83

diff. between two indep. $r$ 's 207

Fisher's transformation 201207

generation of correlated normal r.v.'s 15

significance of $r 37$

test of diff. of $r$ vs. $\rho_{0} 200$

volume (of probability) in the positive

hyperquadrant of a $k$-dimensional

equi-correlated normal distr. 102

and $t$-test for paired means 205

for paired variances 206 
Degrees of freedom $(d f)$

of Chi-square for goodness-of-fit 203

of sample $r 41$

of sample variance 23

Density function

see Probability density function

Distribution

see Probability density function,

Sampling distribution

Distribution function (d.f.) Def. 214

and moments of a distr. 112

Beta rel. with $F 101213$

binomial 165 rel. with $F 58$ with

normal 166 with Poisson 166

C (Cochran's) 121 rel. with $F 122$

Cauchy (as $t_{1}$ ) 39

Chi-square 23-34 rel. with Poisson 219

Dunnett's $t$ 83-84

$\overline{\mathrm{E}}^{2}$ and $\bar{\chi}^{2}$ (monotonic variation) 101

Exponential 215 rel. with $\chi_{2}^{2} 24$

$F v_{1}=v_{2}=1$ and $v_{1}=2, v_{2} \geq 157$ rel. with $t$

57 rel. with $\chi^{2} 57$ rel. with Beta 101

213 rel. with binomial 58

$F_{\max } 111$ rel. with $F 111$

Gamma 216

normal 13

Poisson (rel. with Chi-square) 219 rectangular (discrete uniform) 221

Student's $t 39$ rel. with $F_{1, v} 40$

uniform 221

Dunnett's method 82

Dunn-Šidák criterion 40 example 36

Expectation 215

see Moments

Factorial (function) 215 rel. with Gamma function 215 ascending 215

descending 215
Gamma distribution 216 function 216 rel. with factorial 216

$\mathrm{g}_{1}, \gamma_{1}$ (skewness index) 218-219

$\mathrm{g}_{2}, \gamma_{2}$ (kurtosis index) 218-219

Generation of random variates (r.v.'s) binomial 167-168

Chi-square 25

exponential 24

F 60

normal 14 from random numbers 192 correlated normal 15

Student's $t 41$

uniform 193-194

Harmonic

interpolation see Interpolation

mean of unequal sample sizes $n_{j}$ for

$q$ test 69210

Homogeneity of variances

see Test procedures

HSD (Tukey's method) 67

Integration

analytic, direct 216

numerical 217 trapezoidal rule 217

Simpson's (parabolic) rule 220

Interpolation

for sufficient size $n^{*}(r) 41$

harmonic Def. 217 for $F 453$ for $q 67$

for C 119 for $\ln F 109$

linear Def. 217 for $F 353$ for $q 67$

Isotonic regression

see Regression (monotonic)

Kurtosis $\left(\gamma_{2}, \mathrm{~g}_{2}\right)$ 218-219 
Mean 218 see Moments absolute diff., expect. for X normal 14 of upper $100 \alpha \%$ for X normal 14

Model

Dominance (monotonic variation) Def. 9599 weight function 101 normal 12 for IQ, height, measurement error 12 and goodness-of-fit test 203 Poisson for rare events 219 runs test for residuals with respect to model 182

Simple order (monotonic variation) Def. 9599 weight function 101

Moments Def. 218

Beta 213

binomial 165

calculation by d.f. 112122

C (Cochran's) (partial) 123

Chi $(\chi / \sqrt{ } v) 25$

Chi-square 24

exponential 215

$F$ 59-60 for paired (correlated) variances 60

$F_{\max }$ (partial) 112

Gamma 216

normal 14

number of runs 183-184

Poisson 219

product of two indep. r.v.'s 70

$q$ (partial) 71

rectangular (discrete uniform) 221

sample estimates 219

Student's $t 40$

uniform 221

Multiple comparisons method

general rules viii

Dunnett 82

Newman-Keuls 68

Scheffé 208

Tukey HSD 67
Newman-Keuls' method 68

Permutations

generation of random permutations of numbers 1 to $\mathrm{N} 195$

Probability density function (p.d.f.)

Def. 220

Beta 213

Cauchy (as $t_{1}$ ) 39

Chi $(\chi / \sqrt{v}), s 251 / s 70$

Chi-square 23

exponential 215 rel. with Gamma 215

$F 57$ for paired (corr.) variances 60

Gamma 216 rel. with exponential 216 normal 13

$q 69$

range of $k$ normal r.v.'s 70

Student's $t 38$

uniform 192221

Probability distribution function (d.f.) see Distribution function

Probability mass function (p.m.f.)

binomial 165

number of runs 183

Poisson 219

rectangular (discrete uniform) 192221

Regression

monotonic (isotonic) 95-101

polynomial Def. 140133 conversion

from orthogonal to regular 138-139

runs of positive and negative residuals

182

simple linear by orthogonal 136

test of predicted value from linear

regression 201

Run(s) Def. 181

Sampling (generation of samples)

191 with/without replacement 194-195 
Sampling distribution

of mean 199

of $p$ (proportion) 161-168

of $r 41$

of s.d. $s 25$ of $1 / s 70$

of variance 23

Scheffé's method 208-210

Shape coefficients (of a distribution) see Moments

Simpson's rule 220

see Integration (numerical)

Skewness $\left(\gamma_{1}, g_{1}\right) 218-219$

Standard deviation (s.d.) 221 confidence interval 21 see Moments

Standard error of estimation (based on linear regression) 202 of measurement 12 of a predicted value from linear regression 202

Test procedures

analysis of variance (see Analysis of variance)

for correlation (sample $r$ ) with $\mathrm{H}_{0}: \rho=0$

3741 with $\mathrm{H}_{0}: \rho=\rho_{0} 200$

Dominance model of monotonic variation 98

goodness-of-fit (by Chi-square) 193 202

guessing in a multi-item multiplechoice exam 162

Heads or Tails 162

interaction (dependence) in contingency table 22

mean (sample $\overline{\mathrm{X}}) \quad\left(\sigma^{2}\right.$ known) 199 ( $\sigma^{2}$ unknown) 199
Test procedures (cont.)

number of runs 181-182

power components in polynomial regression 133137

proportion (sample $p$ ) 163

Simple order model of monotonic variation 96

for the diff. between

two error-prone measurements 12

two indep. $r$ coefficients 207

two indep. means $36\left(\sigma_{j}^{2}\right.$ known) 204

two indep. variances 55

two paired means 205

two paired variances 206

means (multiple planned compar.) according to Dunn-Šidák 36

by Dunnett's method 82

means (multiple unplanned compar.)

by Newman-Keuls' method 68

by Tukey's HSD method

by Scheffé's method 208-210 67

homogeneity of variances

$k=2$ variances 55

$k \geq 2 \quad 109-110119207$

rules for multiple comparisons viii

Tukey's HSD method 67

Unequal groups

Bartlett's $\chi^{2}$ 207-208

C $119-120$

Dunnett's $t 83$

$F_{\text {max }} 110-111$

$q$ 68-69

Scheffé's method 210

Variance 222

component of orthogonal polynomial regression 142

confidence interval 21

homogeneity of see Test procedures

see Moments 